\title{
ON NONLINEAR INTEGRODIFFERENTIAL EQUATIONS WITH DEVIATING ARGUMENTS
}

\author{
K. BALACHANDRAN AND S. ILAMARAN
}

\begin{abstract}
We prove an existence theorem for nonlinear integrodifferential equations with deviating arguments using the Schauder fixed theorem.
\end{abstract}

\section{Introduction}

Several authors have studied the problem of existence of solutions of nonlinear integrodifferential equations with deviating arguments $[5,7,8]$. Banaś and Stopka [3] have proved an existence theorem for a differential equation with deviating argument using the measure of noncompactness. Balachandran and Ilamaran [1, 2] have proved existence theorems for nonlinear integral equations with deviating arguments. In this paper we shall derive a set of sufficient conditions for the existence of a solution of integrodifferential equatons with deviatig arguments. The technique used in this paper is similar to that used by Banaś and Stopka [3] and Balachandran and Ilamaran [1, 2].

\section{Basic Assumptions}

Let $p(t)$ be a given continuous function defined on the interval $[0, \infty)$ and taking real positive values. Denote by $C_{p}=C([0, \infty), p(t): R)$ the set of all continuous functions from $[0, \infty)$ into $R$ such that

$$
\sup \{|x(t)| p(t): t \geq 0\}<\infty .
$$

It has been proved [6] that $C_{p}$ forms a real Banach space with regard to the norm

$$
\|x\|=\sup \{|x(t)| p(t): t \geq 0\}
$$

Received January 7, 1994; revised March, 28, 1994.

1991 Mathematics Subject Classification. 45J05, $47 \mathrm{H} 09$.

Key words and phrases. Existence of solutions, nonlinear equations, deviating arguments, Schauder fixed theorem. 
If $x \in C_{p}$ then we will denote $w^{T}(x, \varepsilon)$ the usual modulus of continuity of $x$ on the interval $[0, T]$ i.e.,

$$
w^{T}(x, \varepsilon)=\sup \{|x(t)-x(s)|:|t-s| \leq \varepsilon, t, s \in[0, T]\} .
$$

Our existence theorem is based on the following lemma.

Lemma. [4] Let $E$ be a bounded set in the space $C_{p}$. If all functions belonging to $E$ are equicontinuous on each interval $[0, T]$ and $\lim _{T \rightarrow \infty} \sup \{|x(t)| p(t): t \geq T\}=$ 0 uniformly with respect to $E$, then $E$ is relatively compact in $C_{p}$.

Consider the integrodifferential equation of the form

$$
x^{\prime}(t)=f\left(t, x(H(t)), \int_{0}^{t} K\left(t, s, x^{\prime}(h(s))\right) d s\right)
$$

with the initial condition

$$
x(0)=0 .
$$

If we define $x^{\prime}(t)=y(t)$ then the equation (1) with condition (2) will be transformed into the following functional-integral equation

$$
y(t)=f\left(t, \int_{0}^{H(t)} y(s) d s, \int_{0}^{t} K(t, s, y(h(s))) d s\right) .
$$

Assume the following conditions:

(i). The function $f:[0, \infty) \times R^{2} \rightarrow R$ is continuous and there exist positive constants $A_{1}$ and $A_{2}$ such that

$$
\left|f\left(t, x_{1}, y_{1}\right)-f\left(t, x_{2}, y_{2}\right)\right| \leq A_{1}\left|x_{1}-x_{2}\right|+A_{2}\left|y_{1}-y_{2}\right|
$$

(ii). Let $\Delta=\{(t, s): 0 \leq s \leq t<\infty\}$.

The function $K: \triangle \times R \rightarrow R$ is continuous and there exist continuous functions $m: \Delta \rightarrow[0, \infty), a:[0, \infty) \rightarrow[1, \infty), b:[0, \infty) \rightarrow[1, \infty)$ such that

$$
|K(t, s, y)| \leq m(t, s)+a(t) b(s)|y|
$$

for all $(t, s) \in \triangle$ and $y \in R$.

In order to formulate other assumptions let us define

$$
L(t)=\int_{0}^{t} a(s) b(s) d s, \quad t \geq 0 .
$$

Take an arbitrary number $M>0$ and consider the space $C_{p}$ with $p(t)=\left[a(t) e^{M L(t)+t}\right]^{-1}$. 
(iii). The function $H:[0, \infty) \rightarrow[0, \infty)$ is continuous and $H(t) \geq t$ for all $t \in[0, \infty)$ and the number

$$
m=\sup \left\{e^{-M L(t)} \int_{t}^{H(t)} a(s) e^{M L(s)} d s: t \geq 0\right\}<\infty
$$

(iv). The function $h:[0, \infty) \rightarrow[0, \infty)$ is continuous and $h(t) \leq t$ for all $t \in[0, \infty)$ and there exists a constant $Q>0$ such that

$$
a(h(t)) \leq Q a(t)
$$

(v). There exist constants $B_{1}>0, B_{2}>0$ such that for any $t \in[0, \infty)$ the following inequalities holds

$$
\int_{0}^{t} m(t, s) d s \leq B_{1} a(t) e^{M L(t)}
$$

and

$$
|f(t, 0,0)| \leq B_{2} a(t) e^{M L(t)} .
$$

(vi). $\left[A_{1}\{(1 / M)+m\}+A_{2}\left\{B_{1}+(Q / M)\right\}+B_{2}\right]<1$.

\section{Existence Theorem}

Theorem: Assume that the hypotheses ( $i)$ to (vi) hold. Then the equatoin (2) has at least one solution $y$ in the space $C_{p}$ such that $|y(t)| \leq a(t) e^{M L(t)}$ for any $t \geq 0$.

Proof. Define a transformation $F$ in the space $C_{p}$ by

$$
(F y)(t)=f\left(t, \int_{0}^{H(t)} y(s) d s, \int_{0}^{t} K(t, s, y(h(s))) d s\right)
$$

From our assumptions we observe that $(F y)(t)$ is continuous on the interval $[0, \infty)$. Define the set $E$ in $C_{p}$ by

$$
E=\left\{y \in C_{p}:|y(t)| \leq a(t) e^{M L(t)}\right\}
$$

Clearly $E$ is nonempty, bounded, convex and closed in $C_{p}$. Now we prove that $F$ maps 
the set $E$ into itself. Take $y \in E$. Then from our assumptions we have

$$
\begin{aligned}
|(F y)(t)| \leq & A_{1} \int_{0}^{H(t)}|y(s)| d s+A_{2} \int_{0}^{t}|K(t, s, y(h(s)))| d s+|f(t, 0,0)| \\
\leq & A_{1} \int_{0}^{t} a(s) e^{M L(s)} d s+A_{1} \int_{t}^{H(t)} a(s) e^{M L(s)} d s+A_{2} \int_{0}^{t} m(t, s) d s \\
& +A_{2} \int_{0}^{t} a(t) b(s)|y(h(s))| d s+B_{2} a(t) e^{M L(t)} \\
\leq & \left(A_{1} / M\right) \int_{0}^{t} M b(s) a(s) e^{M L(s)} d s+A_{1} m e^{M L(t)}+A_{2} B_{1} a(t) e^{M L(t)} \\
& +A_{2} a(t) \int_{0}^{t} b(s) a(h(s)) e^{M L(h(s))} d s+B_{2} a(t) e^{M L(t)} \\
\leq & \left(A_{1} / M\right) a(t) e^{M L(t)}+A_{1} m a(t) e^{M L(t)}+A_{2} B_{1} a(t) e^{M L(t)} \\
& +\left(A_{2} Q / M\right) a(t) \int_{0}^{t} M b(s) a(s) e^{M L(s)} d s+B_{2} a(t) e^{M L(t)} \\
\leq & {\left[A_{1}\{(1 / M)+m\}+A_{2}\left\{B_{1}+(Q / M)\right\}+B_{2}\right] a(t) e^{M L(t)} } \\
\leq & a(t) e^{M L(t)}
\end{aligned}
$$

which proves that $F E \subset E$.

Now we want to prove that $F$ is continuous on the set $E$. For this let us fix $\varepsilon>0$ and take $y, z \in E$ such that $\|y-z\| \leq \varepsilon$. Further take an arbitrary fixed $T>0$. In view of (ii) the function $K(t, s, y)$ is uniformly continuous on

$$
[0, T] \times[0, T] \times[-r(h(T)), r(h(T))]
$$

where $r(h(T))=\max \left\{a(h(s)) e^{M L(h(s))}: s \in[0, T]\right\}$. Thus, we have for $t \in[0, T]$

$$
\begin{aligned}
& |(F y)(t)-(F z)(t)| \\
= & \left|f\left(t, \int_{0}^{H(t)} y(s) d s, \int_{0}^{t} K(t, s, y(h(s))) d s\right)-f\left(t, \int_{0}^{H(t)} z(s) d s, \int_{0}^{t} K(t, s, z(h(s))) d s\right)\right| \\
\leq & A_{1} \int_{0}^{H(t)}|y(s)-z(s)| d s+A_{2} \int_{0}^{t}|K(t, s, y(h(s)))-K(t, s, z(h(s)))| d s \\
\leq & A_{1} A H(t) e^{M L(H(t))+H(t)}\|y-z\|+A_{2} \int_{0}^{t}|K(t, s, y(h(s)))-K(t, s, z(h(s)))| d s \\
\leq & A_{1} A B e^{M L(B)+B} \varepsilon+\beta(\varepsilon)
\end{aligned}
$$

where $B=\max \{H(t): t \in[0, T]\}, A=\max \{a(t): t \in[0, B]\}$ and $\beta$ is some continuous function having the property $\lim _{\varepsilon \rightarrow 0} \beta(\varepsilon)=0$. Further, let us take $t \geq T$. Then we have

$$
\begin{aligned}
|(F y)(t)-(F z)(t)| & \leq|(F y)(t)|+|(F z)(t)| \\
& \leq 2 a(t) e^{M L(t)}
\end{aligned}
$$




$$
|(F y)(t)-(F z)(t)| p(t) \leq 2 e^{-t}
$$

Hence for $T$ sufficiently large we have

$$
|(F y)(t)-(F z)(t)| p(t) \leq \varepsilon
$$

By (4) and (5) we get that $F$ is continuous on the set $E$.

Now we prove that $F E$ is relatively compact. For every $y \in E$ we have $F y \in E$ which gives $|(F y)(t)| p(t) \leq e^{-t}$. Hence

$$
\lim _{T \rightarrow \infty} \sup \{|(F y)(t)| p(t): t \geq T\}=0
$$

uniformly with respect to $y \in E$.

Furthermore, let us fix $\varepsilon>0, T>0$ and $t, s \in[0, T]$ such that $|t-s| \leq \varepsilon$ and $s \leq t$. Then for $y \in E$, we have

$$
\begin{aligned}
& |(F y)(t)-(F y)(s)| \\
\leq & f\left(t, \int_{0}^{H(t)} y(u) d u, \int_{0}^{t} K(t, u, y(h(u))) d u\right) \\
& -f\left(t, \int_{0}^{H(s)} y(u) d u, \int_{0}^{s} K(s, u, y(h(u))) d u\right) \mid \\
& +\mid f\left(t, \int_{0}^{H(s)} y(u) d u, \int_{0}^{s} K(s, u, y(h(u))) d u\right) \\
& -f\left(s, \int_{0}^{H(s)} y(u) d u, \int_{0}^{s} K(s, u, y(h(u))) d u\right) \mid \\
\leq & A_{1} \int_{H(s)}^{H(t)}|y(u)| d u+A_{2}\left|\int_{0}^{t} K(t, u, y(h(u))) d u-\int_{0}^{s} K(s, u, y(h(u))) d u\right| \\
& +w^{T}(f, \varepsilon) . \\
\leq & A_{1} A e^{M L(B)} w^{T}(H, \varepsilon)+A_{2}\left|\int_{0}^{t} K(t, u, y(h(u))) d u-\int_{0}^{s} K(t, u, y(h(u))) d u\right| \\
& \left.\left.+A_{2} \mid \int_{0}^{s} K(t, u, y(h(u))) d u-\int_{0}^{s} K(s, u, y) c h(u)\right)\right) d u \mid+w^{T}(f, \varepsilon) \\
\leq & A_{1} A e^{M L(B)} w^{T}(H, \varepsilon)+A_{2} \int_{s}^{t}|K(t, u, y(h(u)))| d u \\
& +A_{2} \int_{0}^{s}|K(t, u, y(h(u)))-K(s, u, y(h(u)))| d u+w^{T}(f, \varepsilon) \\
\leq & A_{1} A e^{M L(B)} w^{T}(H, \varepsilon)+A_{2} \varepsilon \max \left\{m(t, u)+a(t) b(u)[p(h(u))]^{-1}: 0 \leq u \leq t \leq T\right\} \\
& +A_{2} T w^{T}(K, \varepsilon)+w^{T}(f, \varepsilon)
\end{aligned}
$$

which tends to zero as $\varepsilon \rightarrow 0$. Thus $F E$ is equicontinuous on $[0, T]$.

Therefore from the lemma $F E$ is relatively compact. Thus the Schauder fixed point theorem guarantees that $F$ has a fixed point $y \in E$ such that $(F y)(t)=y(t)$. Hence the theorem is proved. 


\section{References}

[1] K. Balachandran and S. Ilamaran, "An existence theorem for Volterra integral equation with deviating arguments," J. Appl. Math. and Stoc. Anal., 3 (1990), 155-162.

[2] K. Balachandran and S. Ilamaran, "Existence of solution for nonlinear Volterra integral equations," Proc. Indian Acad. of Sci.(Math. Sci.), 100 (1990), 179-184.

[3] J. Banaś and Stopka, "The existence and some properties of solutions of a differential equation with deviating argument," Comm. Math. Uni. Carolinae, 22(1981), 525-535.

[4] J. Banaś and K. Goebel, Measure of Noncompactness in Banach Spaces, Marel Dekkar Inc., New York, 1980.

[5] T. A. Burton, Volterra Integral and Differential Equations, Academic Press, New York, 1983.

[6] C. Corduneanu, Integral Equations and Stability of Feedback Systems, Academic Press, New York, 1973.

[7] C. Corduneanu, Integral Equations and Applications, Cambridge University Press, Cambridge, 1991.

[8] G. Gripenberg, S. O. Londen and O. Staffens, Volterra Integral and Functional Equations, Cambridge University Press, Cambridge, 1990.

Department of Mathematics, Bharathiar University, Coimbatore 641 046, Tamil Nadu, India. 